\title{
Relations between the Thermal and Mechanical Properties of Cast Zr-TM-Al (TM: Cu, Ni, or Co) Bulk Glassy Alloys
}

\author{
Yoshihiko Yokoyama ${ }^{1}$, Toru Yamasaki ${ }^{2}$, Peter K. Liaw ${ }^{3}$ and Akihisa Inoue ${ }^{1}$ \\ ${ }^{1}$ Advanced Research Center of Metallic Glasses, Institute for Materials Research, \\ Tohoku University, Sendai 980-8577, Japan \\ ${ }^{2}$ School of Engineering, University of Hyogo, Shosha, Himeji 671-2201, Japan \\ ${ }^{3}$ Faculty of Engineering, The University of Tennessee, 434 Dougherty Engineering Building, \\ Knoxville, Tennessee 37996-2200, USA
}

Ternary Zr-TM-Al (TM: Cu, Ni or Co) bulk glassy alloys were fabricated to clarify the relations between the thermal and mechanical features. These alloy systems show the distinct ternary eutectic point, and a bulk glassy phase also forms around the ternary eutectic composition. We determine the 12 standard Zr-TM-Al bulk glassy alloys in this study. In the twelve standard Zr-TM-Al bulk glassy alloys, the glass-transition temperature has large positive correlation coefficient with Young's modulus and Vickers hardness. The melting temperature has large negative correlation coefficient with Charpy impact value, fracture strain and volume change due to structural relaxation. Furthermore, in order to recognize the origin of ductility, Poisson's ratio (metallic bond nature) and volume change (free volume) were also examined. [doi:10.2320/matertrans.MJ200717]

(Received November 29, 2006; Accepted February 13, 2007; Published June 25, 2007)

Keywords: Zr-TM-Al (TM: Cu, Ni or Co) bulk glassy alloy, Young's modulus, Vickers hardness, tensile fracture strain, Charpy impact value, volume change, Poisson's ratio

\section{Introduction}

Glassy alloys have unique features that result from their glassy random structure and flexible metallic bond. One special trait of glassy alloys is their mechanical properties. Since the glassy alloys exhibit ductile metallic bonding and have a non-periodic structure, we can expect ${ }^{1)}$ extremely high-toughness and high-strength. Some crystalline alloys can exhibit superior plasticity due to systematic dislocation movements. The deformation mechanism of glassy alloys is characterized by the unique adiabatic shear-band movement. ${ }^{2,3)}$ Microscopic fracture-surface images reveal a vein pattern caused by a pseudo-melting state ${ }^{4)}$ in the shear band. Therefore, once a shear band begins to move, it results in a final fracture with the minimal uniform plastic deformation. The lack of the uniform plastic deformability of glassy alloys has been considered to limit the toughness. ${ }^{5}$ )

Ductile glassy alloys, the ductile features of which are derived from the significant elastic strain-energy storage, require the sufficient elastic deformability to avoid the localized stress or strain. A substantial elastic strain, up to $2 \%$ of a glassy alloy, ${ }^{6}$ may be associated with the sufficient stress-relaxation ability to restrict the accidental shear-band activation from a concentrated stress site. Therefore, the ductility of glassy alloys originates from the glass structure. Novikov et al. ${ }^{7)}$ recently described the relationship between the Poisson's ratio and fragility of glass-forming liquids. The Poisson's ratio for bulk glassy alloys, which is influenced by the degree of metallic bond, is usually from $\sim 0.35$ to 0.4 . A high Poisson's ratio is a significant factor to increase the ductility of glassy alloys. This large Poisson's ratio is mainly caused by the volume effect, ${ }^{8)}$ which is closely related to the flexibility of the atomic bond and its configuration. On the other hand, the volume change, ${ }^{9)}$ which is assumed to be the degree of the volume expansion from the fully relaxed state, is also an important factor in estimating the ductility of bulk glassy alloys since it influences the modulus (i.e., Young's modulus) and Poisson's ratio. ${ }^{10)}$ The large value of the volume change means that the expanded glass structure with the excessive open volume, which can relax the localized stress immediately, is also effective to enhance the fracture toughness and fatigue-strength enhancement. ${ }^{11)}$ However, Egami suggested $^{12)}$ that it is not a sufficient consideration to study properties of glassy alloys by the volume effect as exemplified in the free volume theory. We have to consider in detail about the intrinsic difference in the glassy structure. ${ }^{13)}$ Since the thermal and mechanical properties of glassy alloys originate from its own glassy structure and bond state, therefore one can think of some significant relationships between the thermal and mechanical factors. Such significant relationships can derive the pertinent information about the changes in the glassy structure by the compositional or elemental change.

The objective of this paper is to clarify the relationships between the thermal and mechanical properties of $\mathrm{Zr}$-TM-Al (TM: $\mathrm{Cu}, \mathrm{Ni}$ and $\mathrm{Co}$ ) bulk glassy alloys. And we also study the role of the TM element in $\mathrm{Zr}-\mathrm{TM}-\mathrm{Al}$ (TM: $\mathrm{Cu}, \mathrm{Ni}$ and $\mathrm{Co}$ ) bulk glassy alloys.

\section{Experimental Procedure}

Master-alloy ingots of ternary Zr-TM-Al (TM: Cu, Ni and $\mathrm{Co}$ ) alloys were prepared by arc-melting a mixture of pure $\mathrm{Zr}$, $\mathrm{Cu}, \mathrm{Ni}, \mathrm{Co}$ and $\mathrm{Al}$ metals in an argon atmosphere. To maintain a low-oxygen concentration in these alloys, we used a special $\mathrm{Zr}$ crystal rod with oxygen concentration less than 0.05 atomic percent (at\%). $\mathrm{Zr}-\mathrm{Cu}-\mathrm{Al}$ bulk glassy alloys were cast into rod-shaped specimens with $8 \mathrm{~mm}$ in diameter and $60 \mathrm{~mm}$ in length by tilt casting technique. ${ }^{14)}$ In order to estimate the volume change of glassy alloys, densities of the rod-shaped samples $(\phi 8 \times 20 \mathrm{~mm})$ were measured by an Archimedes method at room temperature. The fluid used for 
Table 1 Mechanical and thermal properties of 12 standard Zr-TM-Al (TM $=\mathrm{Cu}, \mathrm{Ni}$ or Co) bulk glassy alloys. CUE is U-notched Charpy impact value, $\sigma_{\mathrm{B}}$ is tensile strength, $\mathrm{HV}$ is Vickers hardness, $E$ is Young's modulus and $\varepsilon_{\mathrm{f}}$ is tensile fracture strain and $\nu$ is Poisson's ratio, $T_{1}$ is liquidus surface temperature, $T_{\mathrm{g}}$ is glass transition temperature, $T_{\mathrm{x}}$ is crystallization temperature.

\begin{tabular}{|c|c|c|c|c|c|c|c|c|c|c|c|}
\hline No & composition & $\Delta V_{\mathrm{r}}$ & $v$ & $\operatorname{CUE}\left(\mathrm{kJ} / \mathrm{m}^{2}\right)$ & $\varepsilon_{\mathrm{f}}(\%)$ & $\mathrm{E}(\mathrm{GPa})$ & $\sigma_{\mathrm{B}}(\mathrm{MPa})$ & $\mathrm{HV}(\mathrm{GPa})$ & $T_{\mathrm{g}}(\mathrm{K})$ & $T_{\mathrm{x}}(\mathrm{K})$ & $T_{1}(\mathrm{~K})$ \\
\hline 1 & $\mathrm{Zr}_{50} \mathrm{Cu}_{40} \mathrm{Al}_{10}$ & 0.41 & 0.363 & 104 & 2.1 & 88 & 1860 & 4.96 & 706 & 792 & 1092 \\
\hline 2 & $\mathrm{Zr}_{52.5} \mathrm{Cu}_{37.5} \mathrm{Al}_{10}$ & 0.40 & 0.364 & 106 & 2.1 & 86 & 1840 & 4.85 & 689 & 779 & 1113 \\
\hline 4 & $\mathrm{Zr}_{50} \mathrm{Cu}_{42.5} \mathrm{Al}_{7.5}$ & 0.30 & 0.366 & 103 & 2.1 & 86 & 1820 & 4.75 & 700 & 771 & 1091 \\
\hline 5 & $\mathrm{Zr}_{55} \mathrm{Cu}_{35} \mathrm{Al}_{10}$ & 0.23 & 0.370 & 99 & 2.2 & 83 & 1810 & 4.70 & 688 & 767 & 1144 \\
\hline 6 & $\mathrm{Zr}_{60} \mathrm{Cu}_{30} \mathrm{Al}_{10}$ & 0.24 & 0.373 & 96 & 2.2 & 80 & 1720 & 4.46 & 671 & 748 & 1151 \\
\hline 7 & $\mathrm{Zr}_{47.5} \mathrm{Cu}_{42.5} \mathrm{Al}_{10}$ & 0.24 & 0.365 & 95 & 2.1 & 90 & 1920 & 5.08 & 718 & 794 & 1114 \\
\hline 8 & $\mathrm{Zr}_{60} \mathrm{Ni}_{25} \mathrm{Al}_{15}$ & 0.31 & 0.371 & 67 & 2.0 & 88 & 1760 & 4.95 & 696 & 787 & 1230 \\
\hline 9 & $\mathrm{Zr}_{65} \mathrm{Ni}_{20} \mathrm{Al}_{15}$ & 0.23 & 0.369 & 60 & 2.0 & 89 & 1780 & 5.02 & 671 & 741 & 1234 \\
\hline 10 & $\mathrm{Zr}_{55} \mathrm{Ni}_{25} \mathrm{Al}_{20}$ & 0.09 & 0.357 & 31 & 1.8 & 99 & 1820 & 5.14 & 733 & 818 & 1305 \\
\hline 11 & $\mathrm{Zr}_{55} \mathrm{Co}_{30} \mathrm{Al}_{15}$ & 0.24 & 0.367 & 32 & 1.8 & 96 & 1750 & 5.30 & 753 & 799 & 1242 \\
\hline 12 & $\mathrm{Zr}_{55} \mathrm{Co}_{25} \mathrm{Al}_{20}$ & 0.13 & 0.356 & 22 & 1.8 & 98 & 1790 & 5.43 & 753 & 823 & 1265 \\
\hline
\end{tabular}

the Archimedes method was the purified water. Thermal factors, such as the glass transition temperature $\left(T_{\mathrm{g}}\right)$ and crystallization temperature $\left(T_{\mathrm{x}}\right)$ of the glassy samples were measured using a differential scanning calorimetry (DSC) in a flowing purified Ar-gas atmosphere with a heating rate of $1.67 \times 10^{-1} \mathrm{~K} / \mathrm{s}$. Liquidus surface temperature $\left(T_{1}\right)$ was obtained from the differential thermal analyzer (DTA) curve, which was obtained by melting the alloy in a purified $\mathrm{Ar}$ atmosphere, and then cooling at a rate of $1.67 \times 10^{-3} \mathrm{~K} / \mathrm{s}$. Both DSC and DTA equipments were manufactured to achieve a high vacuum state under $10^{-3} \mathrm{~Pa}$ before the measurement in Ar gas atmosphere. An X-ray diffractometer was used to examine the crystalline quality and identification of different phases present in the alloys. The tensile strength and Young's modulus were obtained by using an Instron 5582 type test machine. Vickers hardness was measured by using a micro Vickers testing machine by applying a load of $9.8 \mathrm{~N}$ for $15 \mathrm{~s}$. Charpy impact tests were also carried out in air on a $55 \mathrm{~mm}$ long, $10 \mathrm{~mm}$ wide and $5 \mathrm{~mm}$ thick glassy alloy with a U-notch of $2 \mathrm{~mm}$ in depth. All mechanical tests were performed on 10 samples for each alloy composition, and the values of mechanical properties were obtained by averaging the data of 8 samples after removing the maximum and minimum data points.

\section{Results and Discussion}

For the clarification of the major relationship among the mechanical and thermal properties of Zr-TM-Al BGAs, we summarize the mechanical and thermal data in Table 1. In this table, $\Delta V_{\mathrm{r}}$ is the volume change ratio due to fully structural relaxation ${ }^{9)}$ and $v$ is the Poisson's ratio, mechanical properties were represented by the U-notch Charpy impact value (CUE), tensile fracture strain $\left(\varepsilon_{\mathrm{f}}\right)$, Young's modulus $(\mathrm{E})$, tensile strength $\left(\sigma_{\mathrm{B}}\right)$ and Vickers hardness $(\mathrm{HV})$, while thermal properties were represented by the glass transition temperature $\left(T_{\mathrm{g}}\right)$, crystallization temperature $\left(T_{\mathrm{x}}\right)$ and liquidus surface temperature $\left(T_{1}\right)$. About the volume change, $\Delta V_{\mathrm{r}}$, the ternary eutectic point of the alloy numbers 1,8 and 11 shows the maximum value in each alloy system. It has suggested that the ternary eutectic point, whose alloy is characterized by the low melting temperature and crystallization competition, has contributed to freezing the free volume. The component and composition dependabilities of the Poisson's ratio were small, which showed the value about from 0.36 to 0.37 . The Charpy impact value changed drastically with the alloy system or alloy composition, and has suggested the embrittlement accompanying the change of the glass structure. A tensile fracture strain also changes with alloy systems, and it is shown that the change of TM from $\mathrm{Cu}$ to Co has brought about the ductile degradation. Young's modulus shows monotonous compositional dependence, and there is a tendency that it will decrease with the $\mathrm{Zr}$ concentration. The tensile strength does not show the specific composition dependability, but is considered that the compositional dependence will be determined by the abovementioned fracture strain and Young's modulus. Like a Young's modulus, the compositional dependence of hardness decreases monotonically with the $\mathrm{Zr}$ concentration. $T_{\mathrm{g}}$ increases monotonically with $\mathrm{Al}$ and $\mathrm{Cu}$ concentration and its temperature range by compositional change is not so wide. On the other hand, since $T_{1}$ is strongly influenced by a phase diagram, the temperature range by the composition is wide, and the monotonous composition dependability is not shown. Although the $T_{\mathrm{x}}$ shows the similar compositional dependence to $T_{\mathrm{g}}, T_{\mathrm{x}}$ takes maximum value at ternary eutectic temperature and it is not the same at all.

The square of the correlation functions between the mechanical and thermal properties are summarized in Table 2. The thermal factor $T_{\mathrm{g}}$ shows a good correlation with Young's modulus and hardness, whose factors indicate the stiffness of the glass structure. The thermal factor $T_{1}$ exhibits a high correlation with the tensile fracture strain and Charpy impact value, whose factors require a large amount of frozen free volume to relax the localized strain before cracking. Therefore, there are high values of square of correlation factors along the diagonal line in Table 2. Because in mechanical properties in Table 2, the rigidity involves in the upper part as exemplified Young's modulus, and ductility involves in the lower part as exemplified Charpy impact fracture value. However, there are no a good thermal factors with high correlation functions for the tensile strength 
Table 2 Square of correlation function between the thermal and mechanical factors of 12 standard $\mathrm{Zr}-\mathrm{TM}-\mathrm{Al}(\mathrm{TM}=\mathrm{Cu}, \mathrm{Ni}$ or $\mathrm{Co})$ bulk glassy alloys shown in Table 1.

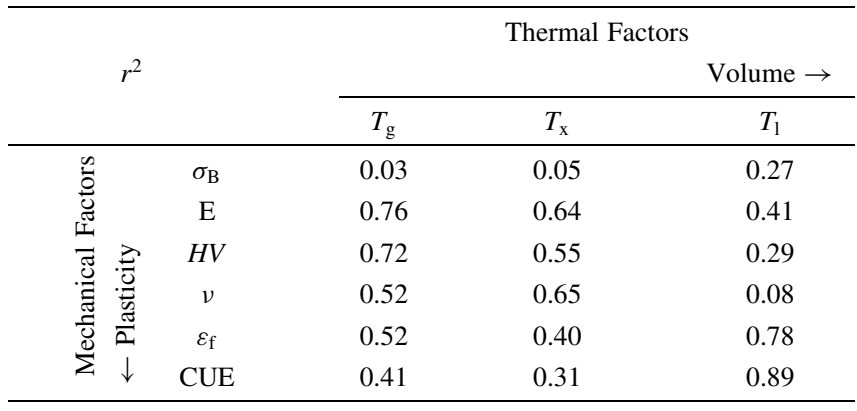

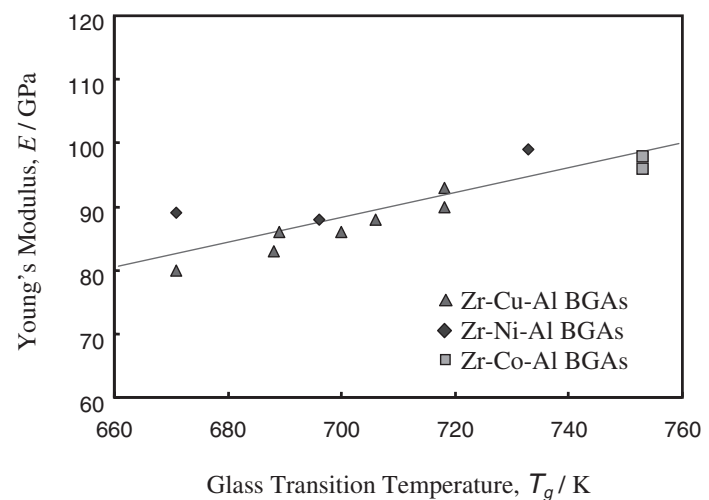

Fig. 1 Relationship between the Young's modulus and glass transition temperature of 12 standard $\mathrm{Zr}-\mathrm{TM}-\mathrm{Al}(\mathrm{TM}=\mathrm{Cu}$, Ni or $\mathrm{Co})$ bulk glassy alloys.

and Poisson's ratio. The Poisson's ratio, which implies degree of metallic bonding, is also shows a monotonic increase with the $\mathrm{Zr}$ concentration.

The difference in TM has brought about the change of the tensile fracture strain $\varepsilon_{\mathrm{f}}$ due to embrittlement, which is decided by the element of TM as shown in Table 1. It is suggested that since the glassy structure has numerous intrinsic glassy structural defects, the degradation of the bonding flexibility in the glassy alloy causes the significant embrittlement. Under compression conditions, in order that a crack may close, the reduction of the yield criterion as fracture strain by the defects is not often seen. The yield criterion, which is equivalent to the tensile strain in tensile test, for indentation deformation could not change among $\mathrm{Zr}$ $\mathrm{TM}-\mathrm{Al}(\mathrm{TM}=\mathrm{Cu}, \mathrm{Ni}$ or $\mathrm{Co})$ glassy alloys on Vickers hardness test. This can be also guessed from the linear relation between the Young's modulus and $T_{\mathrm{g}}$, as shown in Fig. 1. The consideration about the mechanical properties of BGAs, the stiffness of the glass structure is important to cause the strength. Furthermore, the plasticity of the glass is important to cause the enough ductility. The tensile fracture strain is the important factor to consider the yield criterion of BGAs. Figure 2 shows the relationship between the tensile fracture strain and $T_{1}$, and the relationship shows linear relation. The lower $T_{1}$ causes the higher tensile fracture strain. Based on the embrittlement due to the structural relaxation also accompanied with the significant degradation of the tensile fracture strain, the free volume annihilation is probably caused by a significant decrease of the yield

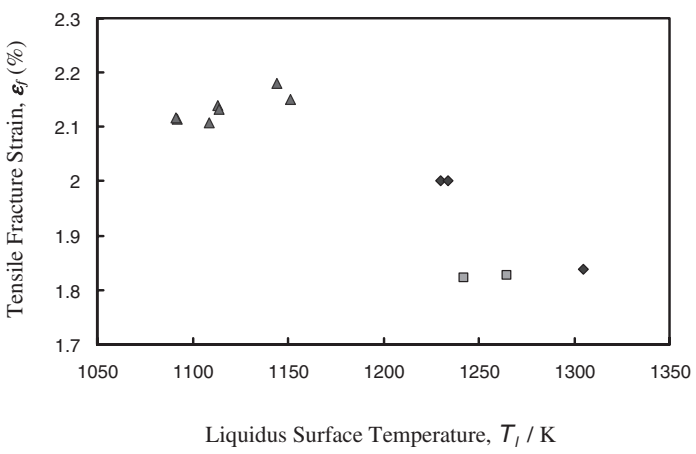

Fig. 2 Relationship between the tensile fracture strain and liquidus surface temperature of 12 standard $\mathrm{Zr}-\mathrm{TM}-\mathrm{Al}(\mathrm{TM}=\mathrm{Cu}, \mathrm{Ni}$ or $\mathrm{Co})$ bulk glassy alloys.

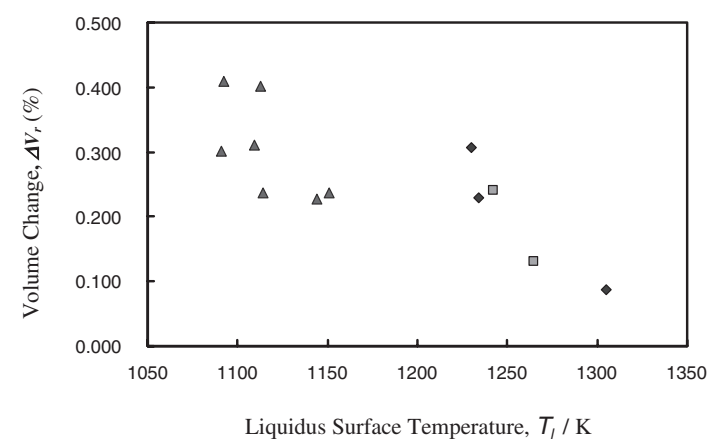

Fig. 3 Relationship between the volume change and liquidus surface temperature of 12 standard $\mathrm{Zr}-\mathrm{TM}-\mathrm{Al}(\mathrm{TM}=\mathrm{Cu}, \mathrm{Ni}$ or $\mathrm{Co})$ bulk glassy alloys.

criterion. Free volume, which considered as open volume in the glass structure, probably acts as the buffer for the atom movement to restrict the fracture. Figure 3 shows the relationship between the volume change and $T_{1}$, and the relationship shows a negative linear relation. Therefore, it is suggested that the higher tensile fracture strain is caused by the higher volume change value. However, in actually, it is not a suitable way to estimate the yield criterion by only the volume change.

The ductility of BGAs is caused mainly by two factors, the metallic bond nature and free volume. The former can be roughly estimated by the Poisson's ratio, and the latter can be estimated by the volume change. We have to make a balance between the Poisson's ratio and volume change in order to obtain a sufficient ductility of BGAs. Since the features of these factors originate from the glassy structure, the atomic arrangement and atomic bond state are probably related to the thermal factors. The Poisson's ratio of glassy alloys, which are about 0.35 to 0.40 , are slightly larger than those of common crystalline alloys. The slightly higher Poisson's ratio of glassy alloys is probably caused by the high degree of the metallic bond nature. However, to stabilize the random glassy structure, the isotropic the metallic bonding may be disadvantageous, and the partial anisotropic bonding is required. The localized electron distribution in metallic bond, which is caused by the chemical interaction among different elements, promotes anisotropic metallic bonding in the glass structure. Especially, the $\mathrm{sp}$-d interaction between $\mathrm{Al}$ (sp-orbit) and $\mathrm{Zr}$ (d-orbit) promotes the strongest atomic 


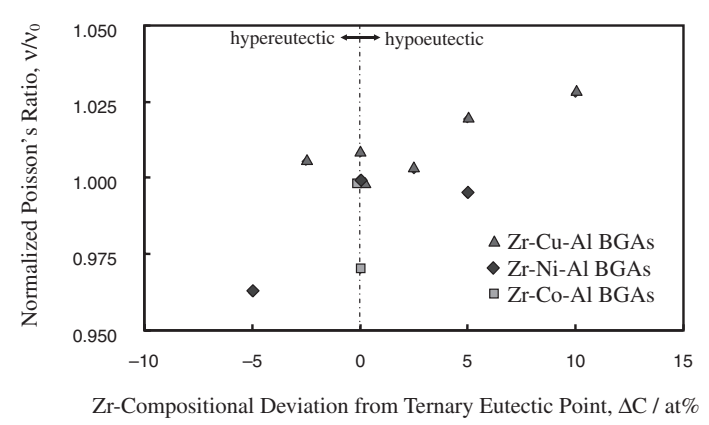

Fig. 4 Relationship between the Poisson's ratio and $\mathrm{Zr}$ concentration, whose values were normalized by the values of ternary eutectic glassy alloys in several alloy systems. $v_{0}$ is the Poisson's ratio value of ternary eutectic glassy alloys.

bond in the $\mathrm{Zr}-\mathrm{TM}-\mathrm{Al}(\mathrm{TM}=\mathrm{Cu}$, Ni or $\mathrm{Co}$ ) alloy system. In order to obtain a ductile glassy alloy with the superior metallic bond nature, the decrease of the sp-d interaction bonds in Zr-TM-Al glassy alloys should be required. Therefore, we can select the solvent $\mathrm{Zr}$ enriched or solute $\mathrm{Al}$ impoverish alloy composition to obtain the ductile glassy alloy with high Poisson's ratio, whereas the glass-forming ability depredates significantly by the higher $T_{1}$. Figure 4 shows the relationship between the Zr content and Poisson's ratio, whose values were normalized by the values of the ternary eutectic glassy alloys in each alloy system. It turns out that Poisson's ratio tends to increase with the solvent $\mathrm{Zr}$ content.

As a result, the volume effect (volume change) is enhanced at the ternary eutectic composition, and the metallic bond nature (Poisson's ratio) is enhanced at the hypoeutectic composition. The ternary eutectic point is characterized by the high stability of the liquid structure even at low temperatures, and the hypoeutectic is characterized by the high stability of the supercooled liquid region. It is difficult to enhance both of Poisson's ratio and volume change by compositional control. However, we can control both the Poisson's ratio and volume change by quaternary additive elements. For example, the $\mathrm{Zr}_{50} \mathrm{Cu}_{37} \mathrm{Al}_{10} \mathrm{Pd}_{3}$ bulk glassy alloy, whose volume change is enhanced up to $0.5 \%$ and Poisson's ratio is also increased up to $0.4,{ }^{15}$ ) exhibits an extremely high fatigue limit over $1 \mathrm{GPa}^{11)}$ Based on this method, we can enhance the ductility of BGAs in other alloy systems in the future.

\section{Summary}

In order to reveal the relationships among the volume, thermal and mechanical features, twelve standard Zr-TM-Al
$(\mathrm{TM}=\mathrm{Cu}$, Ni or $\mathrm{Co})$ bulk glassy alloys were studied. The results obtained are summarized as followings.

(1) The liquids surface temperature has a large correlation coefficient with the Charpy impact value, fracture strain and volume change ratio. $T_{\mathrm{g}}$ has a large correlation coefficient with Young's modulus and Vickers hardness. No suitable thermal factor can be seen to estimate the tensile strength.

(2) In order to obtain a ductile BGA, the volume effect (volume change) is enhanced at the ternary eutectic composition, and the metallic bond nature (Poisson's ratio) is enhanced at the hypoeutectic composition. Therefore, we have to make a balance between them to obtain an inherently ductile glassy alloy.

\section{Acknowledgement}

This research was funded in part by Grant-in-Aid for Scientific Research on Priority Area (Materials Science of Bulk Metallic Glasses) and Grant-in-Aid for Exploratory Research from the Ministry of Education, Culture, Sports, Science and Technology, and NEDO (the New Energy and Industrial Technology Development Organization).

\section{REFERENCES}

1) A. Inoue: Acta Mater. 48 (2000) 279-306.

2) H. S. Chen and T. T. Wang: J. Appl. Phys. 41 (1970) 5338-5339.

3) B. Yang, M. L. Morrison, P. K. Liaw, R. A. Buchanan, G. Y. Wang, C. T. Liu and M. Denda: Appl. Phys. Lett. 86 (2005) 141904-1-141904-3.

4) H. J. Leamy, H. S. Chen and T. T. Wang: Met. Trans. 3 (1972) 699708.

5) C. C. Hays, C. P. Kim and W. L. Johnson: Phys. Rev. Lett. 84 (2000) 2901-2904.

6) J. Kameda: Materials Sci. \& Eng. A 448 (2007) 235-241.

7) V. N. Novikov and A. P. Sokolov: Nature 431 (2004) 961-963.

8) T. Egami and V. Vitek: J. Non-Cryst. Solids $61 \& 62$ (1984) 499-510.

9) Y. Yokoyama, T. Yamasaki, P. K. Liaw, R. A. Buchanan and A. Inoue: J. of Alloys and Compounds 434-435 (2007) 160-163.

10) A. L. Greer: J. Non-Cryst. Solids $61 \& 62$ (1984) 737-748.

11) Y. Yokoyama, P. K. Liaw, R. A. Buchanan, and A. Inoue: Materials Trans. 47 (2006) 1286-1293.

12) D. Srolovits, K. Maeda, V. Vitek and T. Egami: Philos. Mag. A 44 (1981) 847-866.

13) K. Suzuki: Mater. Trans. 39 (1998) 693-699.

14) Y. Yokoyama, K. Inoue and K. Fukaura: Mater. Trans., JIM 43 (2002) 3199-3205.

15) G. Y. Wang, P. K. Liaw, Y. Yokoyama, W. H. Peter, B. Yang, M. Freels, Z. Y. Zhang, V. Keppens, R. A. Buchanan, C. T. Liu and C. R. Brooks: "Influence of Air and Vacuum Environments on Fatigue Behavior of Zr-Based Bulk Metallic Glasses (BMGs)". 12th International Symposium on Metastable and Nano Materials (ISMANAM). Paris, France, 3-7 July 2005 (14). 